\title{
ON THE MULTIPLICITY OF THE SPECTRUM OF THE SPACE OF CUSP FORMS OF $G L_{n}{ }^{1}$
}

\author{
BY J. A. SHALIKA \\ Communicated by C. C. Moore, July 31, 1972
}

In this note I wish to announce certain preliminary results concerning a class of special functions arising in the theory of representations of groups defined over local fields. Before the preparation of this manuscript, the author obtained a copy of the recent paper [4] of I. M. Gelfand and D. A. Kajdan. Several of the results announced here are proved in their paper. I hope to indicate below what I have obtained independently and also what new results I have obtained since the paper of Gelfand and Kajdan became available. I wish to thank A. W. Knapp for the proof of a central lemma stated below.

1. A conjecture. Let $k$ be a global field. Let $\boldsymbol{G}$ be an algebraic group defined over $k$. If $R$ is a commutative $k$-algebra with identity, $\boldsymbol{G}_{R}$ will denote the group of points of $\boldsymbol{G}$ rational over $R$. The notation $\boldsymbol{G}(R)$ will also be used when convenient. $R^{\times}$will denote the unit group of $R$. Let $A$ denote the ring of adeles of $k$. Let $\omega$ be a (unitary) character of $A^{\times}$trivial on $k^{\times}$. For $\boldsymbol{G}=G L_{n}$, let

$$
C_{\omega}={ }^{0} L_{2}\left(G_{A} / G_{k}, \omega\right)
$$

denote the space of cusp forms on $\boldsymbol{G}_{\boldsymbol{A}}$ associated with $\omega$. Let $\hat{\boldsymbol{G}}_{\boldsymbol{A}}$ denote the set of equivalence classes of admissible, irreducible, unitary representations of $\boldsymbol{G}_{A}$. For $\boldsymbol{G}=G L_{n}, \Pi \in \hat{\boldsymbol{G}}_{A}$, let $m_{0}(\Pi, \omega)$ denote the multiplicity with which $\Pi$ occurs in $C_{\omega}{ }^{2}$

In this note, I want to present some evidence in support of the following:

CONJECTURE 1 . For $\Pi \in \widehat{G L}_{n}(A), m_{0}(\Pi, \omega) \leqq 1$.

The conjecture has, of course, been proved for $G L_{2}$. Classically this statement, somewhat reformulated, appears in the well-known works of E. Hecke and H. Maass in their development of the theory of automorphic forms on $G L_{2}$. A systematic and general approach to Hecke theory was carried out in the framework of the theory of representations by $\mathrm{H}$. Jacquet and R. .P. Langlands [11]. The theory was also developed in a general setting by A. Weil [18]. In the work of Jacquet-Langlands, the proof of the simplicity of the spectrum of the space of cusp forms for $G L_{2}$ is reduced,

AMS (MOS) subject classifications (1970). Primary 22E55, 10D20; Secondary 33A75, $46 \mathrm{~F} 05$.

${ }^{1}$ Research partially supported by NSF grant No. 31348 .

${ }^{2}$ For $\operatorname{ch}(k)=0$, it is well known [11] that the assumption $m_{0}(\Pi, \omega)>0$ implies that $\Pi$ is admissible. 
starting with the Fourier series of such a form, to the uniqueness of the "local Whittaker model" for the various representations of $G L_{2}$ over a local field.

In 1962, the uniqueness of, what perhaps should now be called, a Whittaker model for the representations of $G L_{n}$ (more generally Chevalley groups) over a finite field was formulated and proved by I. M. Gelfand and M. I. Graev [2]. Using these results, S. I. Gelfand proved the existence of such a model for the super-cuspidal representations of $G L_{n}$ over a finite field [5].

While attempting to generalize the results of [2] and [5] to local fields, I observed the existence of a natural "Fourier series" for cusp forms on $G L_{n}$. The consideration of such a series was largely motivated by Proposition 3 of [5].

2. The Fourier series of a cusp form. Let $N=N_{n}$ denote the subgroup of super-triangular matrices of $G L_{n}$. Let $\chi$ be a one-dimensional unitary representation of $N_{A}$ such that

(1) $\chi$ is trivial on $N_{k}$,

(2) $\chi$ is "nondegenerate", i.e., the restriction of $\chi$ to a simple root group is nontrivial.

Let $v$ be a place of $k$. Let $k_{v}$ denote the completion of $k$ at $v$.

Let

$$
G_{0}=\prod_{\nu \text { finite }} G L_{n}\left(k_{v}\right)
$$

(restricted product) and

$$
G_{\infty}=\prod_{v \text { infinite }} G L_{n}\left(k_{v}\right) .
$$

Let $C^{\infty}\left(G_{0}\right)$ denote the space of locally constant, complex-valued functions on $G_{0}$ and $C^{\infty}\left(G_{\infty}\right)$ the space of infinitely differentiable functions on $G_{\infty}$. Let $P=P_{n}$ denote the subgroup of $G L_{n}$ consisting of matrices of the form $\left(x_{i j}\right)$ where $x_{n 1}=\cdots=x_{n n-1}=0$. Let $P_{n}^{\prime}$ denote the subgroup of $P_{n}$ defined by the additional restriction $x_{n n}=1$. For $\boldsymbol{G}=G L_{n}$, let ${ }^{0} C^{\infty}\left(G_{A} / P_{k}\right)$ denote the space of $\phi \in C^{\infty}\left(\boldsymbol{G}_{A}\right)$ such that

(3) $\phi(x p)=\phi(x)\left(x \in \boldsymbol{G}_{A}, p \in P_{k}\right)$

and satisfying the additional hypothesis:

(4) if $U$ is the unipotent radical of a proper parabolic subgroup of $G L_{n}$ defined over $k$, then

$$
\int_{U_{\boldsymbol{A}} / U_{\boldsymbol{k}}} \phi(x u) d u^{*}=0 .
$$

(Here $d u^{*}$ is an invariant measure on the homogeneous space $U_{A} / U_{k}$.) For $\gamma \in G L_{r}(A)$, let 


$$
d[\gamma]=\left(\begin{array}{ll}
\gamma & 0 \\
0 & 1_{n-r}
\end{array}\right)
$$

where $1_{n-r}$ is the $(n-r) \times(n-r)$ identity matrix.

Finally, for $\phi \in{ }^{0} C^{\infty}\left(G_{A} / P_{k}\right), \chi$ as above, let

$$
W_{\phi}(x)=\int_{N_{\boldsymbol{A}} / N_{k}} \phi(x u) \chi(u) d u^{*} \quad\left(x \in \boldsymbol{G}_{\boldsymbol{A}}\right) .
$$

Then we have

THeOrem 1. Let $\phi \in{ }^{0} C^{\infty}\left(\boldsymbol{G}_{A} / P_{k}\right)$. Then

$$
\begin{aligned}
\phi(1) & =\sum_{\gamma_{n-1} \in G L_{n-1, k} / P_{n-1, k}^{\prime}} \cdots \sum_{\gamma_{1} \in G L_{1, k}} W_{\phi}\left(d\left[\gamma_{n-1}\right] \cdots d\left[\gamma_{1}\right]\right) \\
& =\sum_{\gamma \in G L_{n-1, k} / N_{n-1, k}} W_{\phi}(d[\gamma]) .
\end{aligned}
$$

Corollary. The map $\phi \rightarrow W_{\phi}$ from ${ }^{0} C^{\infty}\left(\boldsymbol{G}_{\boldsymbol{A}} / P_{k}\right)$ into the space of complex-valued functions on $\boldsymbol{G}_{\boldsymbol{A}}$ is injective.

3. Reduction to the local case. From the results of $\S 1$, following JacquetLanglands, one may readily reduce Conjecture 1 to the uniqueness of a "local Whittaker model". In positive characteristic, this is immediate. In characteristic zero, there are some convergence problems. For this reason, we introduce an appropriate space of distributions. The definition of this space is well-adapted to the infinitesimal description of B. Kostant of class one representations of real Lie groups.

For $\boldsymbol{G}=G L_{n}$, let $C_{c}^{\infty}\left(\boldsymbol{G}_{A}\right)$ denote the set of elements of $C^{\infty}\left(\boldsymbol{G}_{A}\right)$ with compact support. Let $\Pi \in \hat{\boldsymbol{G}}_{A}$. Let $H$ denote the corresponding Hilbert space, with inner product defined by the norm \| \|. Let $\mathscr{D}(\Pi)$ denote the linear subspace of $H$ spanned by elements of the form $\Pi(f) v$ where $f \in C_{\mathfrak{c}}^{\infty}\left(\boldsymbol{G}_{A}\right), v \in H$. Let $\left(\mathfrak{G}\right.$ be the universal enveloping algebra of $G_{\infty}$. $\left(\mathfrak{5}\right.$ acts on $C_{c}^{\infty}\left(G_{\infty}\right)$ (on the left) as differential operators. $(\mathfrak{F}$ acts on $\mathscr{D}(\Pi)$ in such a way that $\left(\mathfrak{b}\right.$ commutes with the natural action of $C_{c}^{\infty}\left(G_{0}\right)$ and so that

$$
\Pi(D \circ f) v=(-1) \Pi(D) \Pi(f) v
$$

for $D \in \operatorname{Lie}\left(G_{\infty}\right), f \in C_{c}^{\infty}\left(G_{\infty}\right)$. Let $K_{0}$ be the maximal compact subgroup of $G_{0}$ defined by the canonical integral structure on $G L_{n}$. Let $\sigma$ be a finitedimensional (unitary) representation of $K_{0}$. Let $E_{\sigma}$ denote the corresponding projection operator on $H$. Then

$$
E_{\sigma} \mathscr{D}(\Pi)=\mathscr{D}_{\sigma}(\Pi) \subset \mathscr{D}(\Pi) .
$$


We topologize $\mathscr{D}_{\sigma}(\Pi)$ by the seminorms

$$
\|v\|_{D}=\|D v\| \quad(D \in \mathfrak{G}, v \in \mathscr{D}(\Pi)) .
$$

This defines a unique topology on $\mathscr{D}(\Pi)=\bigcup_{\sigma} \mathscr{D}_{\sigma}(\Pi)$. Let $K_{\infty}$ be a fixed maximal compact subgroup of $G_{\infty}$. Put $K=K_{0} \cdot K_{\infty}$. Let $H_{K}$ denote the space of $K$-finite vectors in $H$. Then

LEMMA 1. $H_{K} \subset \mathscr{D}(\Pi)$ and is dense in $\mathscr{D}(\Pi)$.

The proof of Lemma 1 is reduced to the case of a connected Lie group by using the theory of semidirect products of G. W. Mackey. The proof then follows immediately from [7, p. 9].

Now let $\mathscr{D}^{\prime}(\Pi)$ denote the topological dual space of $\mathscr{D}(\Pi)$. Let $I$ be an isometry of $H$ into $L_{2}\left(\boldsymbol{G}_{\boldsymbol{A}} / \boldsymbol{G}_{k}, \omega\right)$ commuting with the action of $\boldsymbol{G}_{A}$. Then $I$ maps $\mathscr{D}(\Pi)$ into $C^{\infty}\left(G_{A}\right)$. For $v \in \mathscr{D}(\Pi)$, let $\mathscr{A}(v)=I(v)(1)$. Then

Proposition 1. $\mathscr{A} \in \mathscr{D}^{\prime}(\Pi)$.

Let $\boldsymbol{G}_{A}$ act on $\mathscr{D}^{\prime}(\Pi)$ by duality. Let $\mathscr{D}_{\chi}^{\prime}(\Pi)$ denote the space of $\lambda \in \mathscr{D}_{\chi}^{\prime}(\Pi)$ such that

$$
u \circ \lambda=\chi^{-1}(u) \lambda \quad\left(u \in N_{A}\right)
$$

Define $\lambda_{\mathscr{A}}$ by

$$
\lambda_{\mathscr{A}}(v)=W_{I(v)}(1) \quad(v \in \mathscr{D}(\Pi)) .
$$

COROLlaRY. $\lambda_{\mathscr{A}} \in \mathscr{D}_{\chi}^{\prime}(\Pi)$.

As in [11], one can prove that Conjecture 1 is implied by

CONJECTURE 2 . For $\chi$ nondegenerate, $\Pi \in \widehat{G L}_{n}(A), \operatorname{Dim} \mathscr{D}_{\chi}^{\prime}(\Pi) \leqq 1$.

4. The local Whittaker model. Let $G_{v}=G L_{n}\left(k_{v}\right)$. Let $\hat{G}_{v}$ denote the set of admissible, irreducible, unitary representations of $G_{v}$. For $\Pi \in \hat{G}_{v}$; one can define $\mathscr{D}_{v}(\Pi)$ and $\mathscr{D}_{\chi, v}^{\prime}(\Pi)$ as above. For $\Pi \in \hat{G}_{A}$, write $\Pi$ uniquely in the form $\bigotimes_{v} \Pi_{v}\left(\Pi_{v} \in \hat{G}_{v}\right)$. We have

LEMMA 2. $\bigotimes_{v} \mathscr{D}_{v}\left(\Pi_{v}\right)$ is dense in $\mathscr{D}(\Pi)$.

(Alternatively, one could define $\mathscr{D}(\Pi)$ by this lemma.)

There are two consequences. Firstly,

Proposition 2. Let $\Pi \in \widehat{G L}_{n}(A)$. Suppose $m_{0}(\Pi, \omega)>0$. Then, for all places $v$ of $k, \operatorname{Dim} \mathscr{D}_{\chi, v}^{\prime}\left(\Pi_{v}\right)>0$.

Secondly, one sees immediately that Conjecture 2 is implied by

ConJeCture 3. For all places $v$ of $k$ and all $\Pi \in \widehat{G}_{v}, \operatorname{Dim} \mathscr{D}_{\chi, v}^{\prime}(\Pi) \leqq 1$.

Conjecture (3) has been proved by Gelfand and Kajdan (Theorem 4 of [4]) for nonarchimedean local fields. Hence, if $\operatorname{ch}(k)>0$, it follows, 
immediately from the result of Gelfand and Kajdan and $\S 1$ above, that we have the

THEOREM. Suppose the characteristic of $k$ is positive. Let $\Pi$ belong to $\widehat{G L}_{n}(A)$. Then $m_{0}(\Pi, \omega) \leqq 1$.

In characteristic zero, again by using the results of Gelfand and Kajdan and $\S 1$ and $\S 2$ above, Conjecture 1 is immediately reduced to Conjecture 3 for $G L_{n}(\boldsymbol{R})$ and $G L_{n}(\boldsymbol{C})$. At the present writing, Conjecture 3 is still open for these groups other than for class one representations (see below).

It is of some interest to consider the analogue of Conjecture 3 for a connected, reductive group $\boldsymbol{G}$ defined over $k_{v}$ as well as for connected reductive Lie groups. The conjecture was indeed proved by Gelfand and Kajdan for a suitably restricted class of Chevalley groups over nonarchimedean local fields. For $v$ nonarchimedean, let $K_{v}$ be a "good" maximal compact subgroup of $\boldsymbol{G}_{k_{v}}$ as defined in [16]. In the direction of Conjecture 3 in the general case, one has

Proposition 3. Let $\boldsymbol{G}$ be a connected reductive group and $Q$ be a minimal parabolic subgroup of $\boldsymbol{G}$ both defined over $k_{v}$. Suppose $v$ is nonarchimedean. Let $K_{v}$ be a maximal compact subgroup of $\boldsymbol{G}\left(k_{v}\right)$ as above. Let $\Pi \in \hat{\boldsymbol{G}}\left(k_{v}\right)$. Suppose $\Pi$ has a $K_{v}$ fixed vector. Then there exists a (quasi-) character $\alpha$ of $Q\left(k_{v}\right)$ such that $\Pi_{v}$ is a quotient of ind $\operatorname{in}_{\phi\left(k_{v}\right) \uparrow \boldsymbol{G}\left(k_{v}\right)} \alpha^{3}$

An analogous result for connected semisimple Lie groups was obtained by B. Kostant [13].

For $\Pi$ as in Proposition 3 , the fact that $\operatorname{Dim} \mathscr{D}_{\chi, v}^{\prime}(\Pi) \leqq 1$ is a simple consequence of the method of F. Bruhat [1]. We also have

Proposition 4. Let $G_{1}$ be a connected semisimple real Lie group, $K_{1} a$ maximal compact subgroup. Let $\Pi$ be an irreducible unitary representation of $G_{1}$ having a $K_{1}$-fixed vector. Then $\operatorname{Dim} \mathscr{D}_{\chi}^{\prime}(\Pi) \leqq 1$.

The proof of Proposition 4 may be obtained as a consequence of a theorem of F. Bruhat and the following lemma whose proof was kindly communicated to me by A. W. Knapp.

LEMMA 3. Let $\Pi$ be a representation of the class one principal series of a connected semisimple Lie group $G_{1}$. П acts on $C_{c}^{\infty}\left(K_{1}\right)$. Let $B$ be a formally skew-Hermitian, semidefinite, bilinear form defined on the $K_{1}$-finite vectors in $C_{c}^{\infty}\left(K_{1}\right)$. Then $B$ is continuous in the Schwartz topology.

The proof of this lemma relies on [12], [14] and [17].

\footnotetext{
${ }^{3}$ Since the preparation of this manuscript, the author has been informed that generalizations of Proposition 3 had been obtained independently by W. Casselman, H. Jacquet and Harish-Chandra.
} 
As a consequence of Propositions 3 and 4, one obtains explicit integral representations for the Whittaker functions associated with the class one representations of the groups described in those propositions. This constitutes a step in the direction of explicitly determining the Fourier coefficients of cusp forms attached to $G L_{n}$. These integrals ${ }^{4}$ were studied by $\mathrm{H}$. Jacquet in $[\mathbf{1 0}]$.

Now suppose $v$ is archimedean. As a consequence of Proposition 4, by using the theory of semidirect products of Mackey, one can prove that if $\Pi$ is an irreducible class one unitary representation of $G L_{n}\left(k_{v}\right)$ then $\operatorname{Dim} \mathscr{D}_{\chi, v}(\Pi) \leqq 1$. This result together with Proposition 3 implies

THEOREM 2. Suppose $\Pi \in \widehat{G L}_{n}(A)$ is of class one with respect to $K$. Then $m_{0}(\Pi, \omega) \leqq 1$.

As indicated above, Theorem 2 may be deduced from the work of Gelfand and Kajdan in positive characteristics.

5. Super-cuspidal representations of $G L_{n}$. In this section $v$ will denote a nonarchimedean place of $k$. Let $G_{v}$ denote $G L_{n}\left(k_{v}\right)$.

The following theorem was proved independently by the present author and by Gelfand and Kajdan.

TheOREM 3. Suppose $\Pi$ is a super-cuspidal representation of $G_{v}$. Then $\operatorname{Dim} \mathscr{D}_{\chi, v}^{\prime}(\Pi)=1$.

This result is partially a consequence of the following $L_{2}$ analogue of Theorem 4 of $[4]$.

Proposition 5. Let $\chi$ be a nondegenerate one-dimensional unitary representation of $N_{k_{v}}$. Then the commuting algebra (in the $L_{2}$ sense) of the representation $\operatorname{ind}_{N_{k v} \uparrow G_{v}} \chi$ is commutative.

I have also proved a $C_{c}^{\infty}$ analogue of Proposition 5. This result does not in any way imply Conjecture 3 for nonarchimedean fields. However, one can deduce that $\operatorname{Dim} \mathscr{D}_{x, v}^{\prime}(\Pi) \leqq 1$ for $\Pi$ super-cuspidal. The fact that, in this case, a Whittaker model actually exists was proved by the author by essentially the same method as the proof of Theorem 1. By similar methods, I have also proved that, for all $\Pi \in \hat{G}_{v}$, there exists a unitary character $\chi_{0}$ of $N_{k_{v}}$ (not necessarily nondegenerate) such that $\operatorname{Dim} \mathscr{D}_{\chi_{0}, v}^{\prime}(\Pi) \geqq 1$.

Now, for $f \in C_{c}^{\infty}\left(G_{v}\right)$ and $x \in G_{v}$, define $L_{x} f$ and $R_{x} f$ so that

$$
\begin{aligned}
& \left(L_{x} f\right)(y)=f\left(x^{-1} y\right), \\
& \left(R_{x} f\right)(y)=f(y x) \quad\left(y \in G_{v}\right) .
\end{aligned}
$$

As in [2], [4], define an involution $\theta$ on $G_{v}$ by

\footnotetext{
${ }^{4}$ See also the references given in $[\mathbf{1 0}]$.
} 


$$
\theta(x)=w_{0}{ }^{t} x w_{0} .
$$

Here $w_{0}$ denotes the $n \times n$ matrix with 1 along the antidiagonal and zero elsewhere. ${ }^{t} x$ denotes the transpose of the $n \times n$ matrix $x$. Proposition 5 is a consequence of

Proposition 6. Let $T$ be a distribution on $G_{v}$ satisfying $T\left(L_{u_{1}-1} f R_{u_{2}}\right)$ $=\chi\left(u_{1} u_{2}\right) T(f)$ for all $f \in C_{c}^{\infty}\left(G_{v}\right), u_{1}, u_{2} \in N_{k_{v}}$. Then

$$
T\left(f^{\theta}\right)=T(f)
$$

for all $f \in C_{c}^{\infty}\left(G_{v}\right)$.

This proposition was proved independently by the present author and by Gelfand and Kajdan.

Since the writing of the first draft of this manuscript, the author succeeded in proving the analogue of Proposition 6 for $v$ archimedean, provided $T$ is a measure. At the present writing, $I$ have not been able to extend this latter result to a general distribution. However, I have been able to show, by using the method of proof of Theorem 4 of [4], that the analogue of Proposition 6 for $v$ archimedean and general $T$ implies Conjecture $1 .^{5}$

6. $L$-functions. Proceeding by analogy with the definition of the Gauss sums defined by Gelfand and Kajdan, I have defined a natural $L$-function associated with a pair of cusp forms associated to class one representations of the groups $G L_{n}$ and $G L_{n-1}$ respectively. These $L$ functions are defined by everywhere convergent integrals as in the work of Jacquet-Langlands. The proof of the functional equation for these $L$-functions may be obtained by analogy with the method of the paper of Gelfand and Kajdan. By using Theorem 1 of the present paper, one can show that these $L$-functions are given by Euler products, provided these products converge. As in Jacquet-Langlands, the local Euler factors may be expressed as nonexplicit integrals involving pairs of Whittaker functions. It is reasonable to expect that explicit expressions for these Euler factors (in the class one case) can be obtained by a further study of Whittaker functions. I hope that Propositions 3 and 4 above will be useful in this direction.

\section{BIBLIOGRAPHY}

1. F. Bruhat, Sur les représentations induites des groupes de Lie, Bull. Soc. Math. France 84 (1956), 97-205. MR 18, 907.

2. I. M. Gel'fand and M. I. Graev, Construction of irreducible representations of simple algebraic groups over a finite field, Dokl. Akad. Nauk SSSR 147 (1962), 529-532= Soviet Math. Dokl. 3 (1962), 1646-1649. MR 26 \#6271.

\footnotetext{
${ }^{5}$ Since the preparation of this manuscript, I have succeeded in proving Proposition 6 for $v$ archimedean, provided $T$ is an eigendistribution for the Casimir operator associated with $G_{v}$. This result is sufficient to prove the conjectures of this paper.
} 
3. I. M. Gel' fand, M. I. Graev and I. I. Pjateckiī-Šapiro, Theory of representations and automorphic functions, "Nauka”, Moscow, 1966; English transl., Saunders, Philadelphia, Pa., 1969. MR 36 \# 3725; MR 38 \# 2093.

4. I. M. Gel'fand and D. A. Kajdan, Representations of $G L(n, K)$ where $K$ is a local field, Institute for Applied Mathematics, No. 942, 1971.

5. S. I. Gel'fand, Analytic representations of the full linear group over a finite field, Dokl. Akad. Nauk SSSR 182 (1968), 251-254= Soviet Math. Dokl. 9 (1968), 1121-1125. MR 38 \#1188.

6. R. Godement, Notes on Jacquet-Langland's theory, Institute for Advanced Study, 1970.

7. Harish-Chandra, Discrete series for semisimple Lie groups. II. Explicit determination of the characters, Acta Math. 116 (1966), 1-111. MR 36 \#2745.

8. - Harmonic analysis on reductive p-adic groups, Lecture Notes in Math., no. 162, Springer-Verlag, Berlin and New York, 1970. (Notes by G. Van Dijk.)

9. , Representations of a semisimple Lie group on a Banach space. I, Trans. Amer. Math. Soc. 75 (1953), 185-243. MR 15, 100.

10. H. Jacquet, Une interprétation géométrique et une généralisation p-adique des fonctions de Whittaker en théorie des groupes semi-simples, C. R. Acad. Sci. Paris Sér. A-B 262 (1966), A943-A945. MR 34 \# 285.

11. H. Jacquet and R. P. Langlands, Automorphic forms on GL(2), Lecture Notes in Math., no. 114, Springer-Verlag, Berlin and New York, 1970.

12. A. W. Knapp and E. M. Stein, Intertwining operators for semi-simple groups, Ann. of Math. (2) 93 (1971), 489-578.

13. B. Kostant, On the existence and irreducibility of certain series of representations, Bull. Amer. Math. Soc. 75 (1969), 627-642. MR 39 \#7031.

14. - Irreducibility of the principal series and existence and irreducibility of the complementary series, Lecture Notes, Advanced Science Seminar, Bowdoin College, Brunswick, Me., 1968.

15. I. G. Macdonald, Spherical functions on a $\mathscr{P}$-adic Chevalley group, Bull. Amer. Math. Soc. 74 (1968), 520-525. MR 36 \# 5141.

16. I. Satake, Theory of spherical functions on reductive algebraic groups over $\mathscr{P}$-adic fields. I, Inst. Hautes Études Sci. Publ. Math. No. 18 (1963), 5-69. MR 33 \# 4059.

17. G. Schiffmann, Intégrales d'entrelacement, C. R. Acad. Sci. Paris Sér. A-B 266 (1968), A47-A49. MR 38 \#2248.

18. A. Weil, Dirichlet series and automorphic forms, Lecture Notes in Math., no. 189, Springer-Verlag, Berlin and New York, 1971.

Department of Mathematics, The Johns Hopkins University, Baltimore, MaryLAND 21218 